\title{
CORRIGENDUM
}

\section{Prognostic impact of iron parameters in patients undergoing allo-SCT}

GN Bazuaye, A Buser, S Gerull, A Tichelli and M Stern

Bone Marrow Transplantation (2012) 47, 616; doi:10.1038/bmt.2012.47

Correction to: Bone Marrow Transplantation (2012) 47, 60-64; doi:10.1038/bmt.2011.13

Since the publication of this article, the authors have noticed that GN Bazuaye's name was spelt incorrectly. The correct authors list is shown above.

The authors would like to apologize for the inconvenience caused. 